\title{
Are mega-events a solution to address physical inactivity? Interrogating the London 2012 Paralympic sport participation legacies among people with disabilities
}

\begin{abstract}
Understanding why non-active people with disabilities have not participated in more sport since the London 2012 Paralympic Games is the focus of this paper. This research reviews the constraints preventing people with disabilities from participating in more sport, and the role the London 2012 Paralympic Games plays in this. 81 people with disabilities in England who are non-active completed an online questionnaire exploring their constraints to participating in more sport. The data suggests attitudes towards the London 2012 Paralympic Games were not an important reason for the lack of sport participation: instead, systemic and social barriers are more constraining to participation in more sport. A factor analysis identified four components that constrain non-active people with disabilities: sport provision; economic; unawareness of how to include people with disabilities in sporting activities; and access to sport participation opportunities. The four components explained $72.93 \%$ of the total variance. It is recommended mega sporting events are not used as a policy intervention to increase sport participation of people with disabilities, as this does not account for constraining social and systemic barriers to sports participation. Instead, bottom-up solutions designed and managed in conjunction with people with disabilities may be more effective.
\end{abstract}

\section{Introduction}

Governments often believe the hosting of a mega sporting event (MSE), such as the Olympic Games or Paralympic Games, will lead to increased grassroots sport participation (Bloyce \& Lovett, 2012; Grix \& Carmichael, 2012). It is suggested MSEs are 'mythopoeic' in character, whereby claims about sport participation as a result of MSEs may have some elements of truth but the impact of MSEs on sport participation have been distorted by myth, which is largely unexamined (Coalter, 2007). The mythopoeic qualities of MSEs can be seen by the United Kingdom's (UK) government's decision to place policy emphasis on the London 2012 Olympic and Paralympic Games increasing sport participation. Indeed, a pledge of getting one million more people active as a result of the London 2012 Games was triumphantly pronounced as part of the fanfare surrounding claims about what the Games might do for people's relationship with sport (DCMS, 2008). Lord Sebastian Coe, as part of the London 2012 bid team, persuaded the International Olympic Committee to choose London as the host for the 2012 Olympic and Paralympic Games, largely thanks to the promise of a significant legacy for sport (Gibson, 2009). In 2007, the UK government released its legacy pledges for the Olympic and Paralympic Games (DCMS, 2008), though there was a lack of attention paid to how legacies from the Paralympic Games, specifically, were going to be achieved (Weed \& Dowse, 2009). This prompted accusations the legacy potential of the Paralympic Games was not being explored in enough detail (Weed \& Dowse, 2009). The apparent side-lining of the legacy preparations for the Paralympic Games was likely because the Paralympic Games is hosted after the Olympic Games because of contractual obligations (Cashman, 2006); the Paralympic Games is rarely a primary motive for bidding to host the Games (Darcy \& Appleby, 2011). Following the criticism for a lack of specific legacy plans (Weed \& Dowse, 2009), three general 
objectives for the London 2012 Paralympic Games (LPG) legacy were articulated in the legacy strategy, 'London 2012: A legacy for disabled people'. These were to:

1) affect a positive attitudinal change in how disabled people are viewed in society;

2) to increase the opportunities for disabled people to participate in sport and to be physically active, and

3) to increase the opportunities for disabled people in employment (DCMS, 2010).

The Coalition government ${ }^{i}$ did update Labour's 2010 legacy pledges in April 2011 but increasing the sport participation of people with disabilities (PWD), regardless of life-stage, was kept as a legacy aim (Office for Disability Issues, 2011). The UK government was clearly using the LPG as a policy vehicle for increasing the sports participation of PWD (Gibson, 2012).

The purpose of this study is twofold. First, this research aims to understand why non-active PWD have not participated in more sport since the London 2012 Paralympic Games (LPG), therefore building on Brown and Pappous' (2018a) investigation into the declining sport participation of PWD after the LPG in England. Second, this research intended to discover the influence of attitudes towards the LPG from non-active PWD as part of overall constraints to participating in more sport To improve sport participation policy for this population group in the future, it is important to understand the main constraints to regular sport participation. If attitudes towards mega sport events, in this case the LPG, proved to be an important constraint, understanding the ways in which the reactions to hosting the LPG prevented more sport participation will help future governments aiming to use MSEs as part of their sport participation policy. Consequently, this study is guided by the following two research questions:

- What are the main constraints preventing non-active PWD from participating in more sport?

- What role did attitudes towards the LPG play in constraints to more sport participation?

To help us position this research within the wider literature, we first review the barriers and constraints to sport participation for PWD. We then discuss the main theories and evidence for sport participation from the Paralympic Games.

\section{Barriers to sports participation for PWD}

Generally, PWD participate in sport less often and less frequently than people without disabilities (ECORYS, 2018). In England, reducing the gap in participation between PWD and people without disabilities has been recognised as an important strategic priority for the UK government (HM Government, 2015; Sport England, 2016). Since the LPG in 2012, Sport Englandii has significantly increased its investment in disability sport to reduce the participation gap (Brown, 2019)iii. Despite the comparatively low levels of sport participation, there is evidence to suggest lower participation is not due to a lack of desire to be physically active (Activity Alliance, 2020). While intrinsic motivation can be a barrier for some individuals (Perreault \& Vallarand, 2007; Scelza, Kalpakjian, Zemper \& Tate, 2005), there must also be other barriers hindering increased sport participation. For some individuals, their impairment can be a constraint to sports participation (Activity Alliance, 2020; Jaarsma, Dijkstra, Geertzen \& Dekker, 2014), with the salience of this constraint differing depending on the nature of the impairment (Darcy, Lock \& Taylor, 2017). Individuals with severe 
impairments may require a greater level of support to participate in certain sporting opportunities and providing the right level of support can be a barrier for some sport providers and individuals (Darcy, Lock \& Taylor, 2017). Environmental constraints can also hinder some PWD from participating in sport (Darcy, Lock \& Taylor, 2017; Jaarsma et al., 2014). Difficulties accessing appropriate transport and availability of suitable sporting facilities have been claimed to constrain participation for some PWD (Brown, 2019; Darcy, Lock \& Taylor, 2017; Jaarsma et al., 2014; Ives, Clayton, Brittain \& Mackintosh, 2019). Research carried out in Australia found five structural constraints to participation - support from the community or organisation, time available for sport participation, cost and availability of sports equipment, economic challenges, location of sport setting and transport considerations - as well as interpersonal and intrapersonal, as constraints to sport participation (Darcy, Lock \& Taylor 2017). The authors discovered the level of support needs for the disabled individual is the most likely factor limiting participation in sport (Darcy, Lock \& Taylor 2017).

Cost of sports participation can constrain some PWD (Darcy, Lock \& Taylor, 2017), a barrier likely to be exaggerated for PWD as there is often an inequality in wealth between PWD and non-disabled people (McKnight, 2014). In the UK, austerity measures implemented during the Coalition and Conservative governments reduced the welfare and public services available to PWD, resulting in serious negative consequences for some individuals (Brittain \& Beacom, 2016; Cross, 2013). The economic challenges brought about by austerity, along with negative media characterisations of PWD prevalent during this time-period (Crow, 2014; Briant, Watson, \& Philo, 2013), led to some PWD reducing their sport participation because of a fear of losing their state benefits if seen to be active (Activity Alliance, 2020; Brown \& Pappous, 2018a, 2018b; Christiaens, 2018; Johnson \& Spring, 2018). This is especially problematic and a significant constraint for individuals reliant or dependent to a strong degree on state financial support (Brittain \& Beacom, 2016; Cross, 2013).

Ableism, the prioritisation of non-disabled experiences and perceived norms and standards to the detriment of difference (Campbell, 2001), can be a significant constraint for some PWD. Ableism differs from disablism by describing societal-wide oppression, whereas disablism refers to individual discrimination on the basis of disability (Bogart \& Dunn, 2019). Indeed, ableism has been claimed to reduce a PWD's consumption of sports participation through a failure of the individual generating sufficient reserves of social, economic, and cultural capitals and the ability to self-determine (Brittain, Biscaia \& Gérard, 2020). In England, some sporting organisations have been claimed to have either exhibited direct or indirect ableism through a failure to plan and structure their activities with PWD in mind (Brown \& Pappous, 2018a; Christiaens, 2018; Johnson, 2019). This has led to a lack of suitable sporting opportunities to suit the motivations of some PWD to participate in sport (Ives et al., 2019), as well as some non-disabled coaches being uncomfortable including PWD in mainstream and inclusive sport participation settings (Johnson, 2019). Additionally, a lack of awareness and knowledge of sport participation activities by PWD can constrain sports participation (Ives et al., 2019; Jaarsma et al., 2014; Jaarsma, Haslett \& Smith, 2018). This is particularly problematic as physical activity and sports participation messaging has been demonstrated to be important to an individual's activity levels (Williamson, Baker, Mutrie, Niven \& Kelly, 2020). Messaging for PWD may not be commensurate with important values for PWD (Activity Alliance, 2020), fail to emphasise the fun element of sport (Ives et al., 2019), or be delivered through the right communication channels (Activity Alliance, 2020a; Christiaens, 2018; Ives et al., 2019). 
Having provided a brief overview of some of the barriers potentially constraining sports participation of PWD, the next section will review theories and evidence for the ability of the Paralympic Games to increase the sports participation of PWD.

\section{How effective is the Paralympic Games at increasing sport participation?}

It is thought MSEs may increase sport participation due to the demonstration effect: "...a process by which people are inspired by elite sport, sports people or sports events to participate themselves" (Weed, 2009, p. 4). MSEs might be able to increase the interest to watch sport and contemplation to participate in sport (Grix \& Carmichael, 2012; Lyle, 2009), but there is no evidence to suggest this heightened interest is converted into participation (Lyle, 2009). The possibility of increased desire to participate in sport is likely to appeal to individuals who are already predisposed to being 'sporty' (Grix \& Carmichael, 2012; Lyle, 2009). Moreover, MSEs may deter some people from participating in sport because of a perceived competency gap between the individual and the elite sportsperson (Grix \& Carmichael, 2012; Lyle, 2009). This is because an individual's self-efficacy could be reduced if a comparison between one's own ability compared to the elite athlete is made and judged to be too big to reconcile (Payne, Reynolds, Brown \& Fleming, 2003). The demonstration effect, if it exists, is likely to be potential that needs to be exploited (Weed et al., 2015).

Few studies have specifically investigated how the Paralympic Games may be able to increase the sports participation of PWD (Misener, Darcy, Legg \& Gilbert, 2013; Pappous \& Brown, 2018). Pappous and Brown (2018) were unable to provide robust empirical support for evidence demonstrating the ability of the Paralympic Games to increase participation. Instead, evidence provided for increased sport participation is often anecdotal. For example, it was claimed by the Paralympian, Dame Sarah Storey, more PWD were participating in sport (BBC Sport, 2013), but there was a lack of evidence to substantiate the claims. Similarly, Coward and Legg (2011) offered tentative evidence of the 2010 Vancouver Winter Paralympics being able to provide a pathway for increased sport participation but, like Storey (BBC Sport, 2013), there was a lack of empirical evidence to corroborate the claims. For the LPG, a year after the Paralympics, eighty-nine per cent of sports clubs in the UK had indicated there had been no discernible change in their disabled membership (Sport and Recreation Alliance, 2013). Moreover, research by the EFDS ${ }^{\text {iv }}$ highlighted disability specific clubs in the UK were less likely to have experienced a change in their membership following the London 2012 Games (EFDS, 2013). Brown and Pappous (2018a) add further weight to the lack of sustained impact of the LPG on the sports participation of PWD by explaining how participation declined five years since the event in 2012. The authors highlight a complex combination of social and systemic barriers contributing to the decline in participation, such as a lack of leveraging, austerity measures, and ableist practices (Brown \& Pappous, 2018a).

The Paralympic Games has been referred to as 'disempowering' by some scholars. The diversity of disability is underrepresented in the Paralympic Games, providing a mainstream audience with an unbalanced perception of disability and how it manifests itself for the disabled individual (Braye, Dixon \& Gibbons, 2013; Purdue \& Howe, 2012). The media representation of the Paralympic Games, particularly Paralympians as 'superhumans', has been cited as contributing to the sense of the Paralympic Games as being less inspirational than claimed in the mainstream media (Howe \& Silva, 2018; Purdue \& Howe, 2012). In the past, Paralympians have tended to be characterised in a manner consistent with medicalised narratives of disability (Braye, Dixon \& Gibbons, 2013; Thomas \& Smith, 2003) and a source of pity (Brittain, 2012). In the media, Paralympians have frequently been portrayed as 'supercrips' (Crow, 2014; Gilbert \& Schantz, 2012; Howe, 2011; Silva \& Howe, 2012). The 'supercrip' concept can be 
defined as a '...a stereotyping process that requires an individual "to fight against his/her impairment" in order to overcome it and achieve unlikely "success"' (Silva \& Howe, 2012, p. 175). While some PWD may view the supercrip label as inspirational (Berger, 2008; Silva \& Howe, 2012), most scholars argue the term is a source of disempowerment for 'everyday' PWD, as most PWD are unable to live up to this distorted and unrealistic representation of disability (Crow, 2014; Gilbert \& Schantz 2012; Howe, 2011; Silva \& Howe, 2012). Indeed, for individuals with impairments not included in the Paralympic Games, the capacity for inspiration is undermined (Howe \& Silva, 2018; Purdue \& Howe, 2012), especially as Paralympians with severe impairments failing to adhere to the supercrip narrative receive less media coverage (Gilbert \& Schantz, 2012a; Howe, 2011). Due to the lack of diversity and underrepresentation of disability included in the Paralympic Games, most PWD are ineligible for the Paralympic Games (Howe \& Silva, 2018). Thus, nations and societies attempting to use the Paralympic Games as a way of increasing participation are likely to be frustrated because of the event's exclusivity of disability: it is not inclusive of the wider disability experience for most PWD (Howe \& Silva, 2018).

Having reviewed the literature on barriers to sports participation for PWD and the ability of the Paralympic Games to increase participation, the next section introduces the theoretical framework for the research: the realist evaluation method.

\section{Theoretical framework: realist evaluation}

Realist evaluation is a theory-driven approach to evaluating social programmes and is guided by a basic formula guiding its orientation: context + mechanism $=$ outcome (Pawson \& Tilley, 1997). This formula states that given the right context, allied to the necessary mechanisms, the programme outcomes will be achieved. Programmes are thought to be the sum of participants' reasoning in response to the programme resources that are intended to provoke behaviour change (Astbury, 2013; Pawson \& Tilley, 1997, 2004). The LPG can be viewed as a social programme because one of the main aims of the LPG was to increase the sport participation of PWD (Office for Disability Issues, 2011). Thus, the LPG was viewed as a form of intervention to positively change the health of PWD through increased sport participation (Hughes, 2013). Viewed as a social programme, the LPG can be considered the total sum of the reasoning of PWD in response to resources provided by the LPG designed to increase PWD's grassroots sport participation.

Realist evaluations begin with an identification of the underlying programme theory: the rationale behind the programme (Pawson \& Tilley, 2004). For the LPG, the underlying programme theory was heavily reliant on the demonstration effect. Bloyce and Lovett (2012) conducted a figurational analysis on the legacy documents of the 2012 Olympic and Paralympic Games and discovered inspiration to be the main theme behind how increased sport participation would be achieved. Furthermore, Hughes (2013) identified the demonstration effect and the abstract notion of inspiration as being the hypothesised programme theory. The specific legacy documents for the LPG underline the reliance on inspiration and the demonstration effect (Bloyce \& Lovett, 2012; Hughes, 2013). The programme theory, as suggested in the legacy plans (DCMS, 2010; Office for Disability Issues, 2011), is offered below:

Increased sport participation will be achieved as a result of the inspiration derived from the achievements of Great British Paralympians at the LPG. The inspiration generated by the Great British Paralympians will increase the self-efficacy and motivation of $P W D$ to participate in sport, thus prompting participation in sport. 
The realist evaluator investigates the mechanisms and contexts behind the programme (Pawson $\&$ Tilley, 1997). Mechanisms are not the programme activities, but the interaction between participants' reasoning to the resources offered by a programme (Astbury \& Leeuw, 2010). Mechanisms are always present; whether they are dormant or active depends on the contextual conditions (Pawson \& Tilley, 1997; Pawson, 2013). Social programmes only work because mechanisms 'fire' in the necessary contextual conditions (Pawson \& Tilley, 1997, 2004). Context refers to the conditions needed in order for the programme mechanisms to be triggered (Tilley, 2000). Programme mechanisms enter a social system comprising pre-existing mechanisms and contexts (Pawson \& Tilley, 1997). For the LPG, the contextual conditions and factors to be aware of are considerably complex. As the right to host the Games was confirmed in 2005 and the seven-year gap between the delivery of the Paralympics, there is clearly a great deal of contextual conditions that would have been reproduced and transformed during the build-up to the LPG in relation to the programme mechanisms. In addition, the programme mechanisms of the LPG during the event, and subsequently after the event, will have been of a complex and emergent quality too.

It is understanding why and in what circumstances a social programme works, that is the thrust of the realist evaluation (Pawson, 2013; Pawson \& Tilley, 2004). Thus, context-mechanismsoutcome configurations (CMOCs) provide the realist evaluator with the ability to explain how the programme has worked, for whom, in what circumstances, and how (Pawson \& Tilley, 1997, 2004).

\section{Method}

This section explains the process by which the data was collected to explore the constraints to sport participation of non-active PWD, offering insight into why these individuals have not participated in more sport as was hoped for following the LPG (Brown \& Pappous, 2018a).

\section{Population and sampling strategy}

An online questionnaire was designed and hosted using Bristol Online Survey's (BOS) tool. Disabled people's organisations (DPOs) acted as gatekeepers enabling access to non-active PWD. Only DPOs listed as members of the pan-disability rights organisation, Disability Rights UK (DRUK), were included. This central source was convenient in being able to contact a large number of DPOs, and also acted as a source of quality control, ensuring only recognised DPOs affiliated to DRUK were included. 372 English DPOs were listed as members of DRUK (correct as of 06.10.2017). Of the 372 English DPOs, 164 DPOs had publicly available contact details.

The study was focused on exploring reasons for the lack of sustained sports participation following the LPG, as suggested by Brown and Pappous' study (2018a) and national data from Sport England's Active People Survey ${ }^{v}$ (Active People Interactive, 2017). To build upon Brown and Pappous' study (2018a) and to be consistent with the findings from the Active People Survey data, only individuals aged 16+ who were either inactive or fairly active were eligible for the study. The definitions of inactive and fairly active were taken from Sport England's 'Active Lives' report. Inactivity is defined as being 'less than 30 minutes a week' of activity, whilst individuals who are fairly active are active between '30-149 minutes a week' (Sport England, 2017, p. 3). Inactive or fairly active individuals with disabilities aged 16+ were therefore the population of interest. It was not possible to determine a population figure and response rate. The findings are thus descriptive and provide an insight into constraints for PWD. 
Electronic snowball sampling (Veal \& Darcy, 2014) was used to contact eligible DPOs. The DPOs, if they agreed to participate, contacted their membership promoting the study and the link to completing the questionnaire. Data collection commenced on $5^{\text {th }}$ December 2017 and concluded on the $13^{\text {th }}$ February 2018 at 10pm. In total, 26 of the 164 DPOs confirmed they had sent the questionnaire to their membership, representing approximately $16 \%$ of the contacted DPOs. The final sample for the study was 81 adults with disabilities. Though the final sample might appear low, there have been few studies to collect empirical data on the impact of the Paralympic Games on sports participation, generally (Misener et al., 2013; Pappous \& Brown, 2018). To the authors' knowledge, no other studies have reported the views of non-active PWD and their responses to the Paralympic Games as part of their limited sport participation habits. Furthermore, inactive or less active adults with disabilities can be considered a hard to access population group (Bonevski et al., 2014; Rockliffe, Chorley, Marlow \& Forster, 2018). It is therefore argued a sample of 81 represents an adequate sample for obtaining an insight into the experiences of this population after the LPG, as little data for this population currently exists.

\section{Research instrument}

The purpose of the study was to gain an insight into why non-active PWD have not participated in more sport since the LPG. Consequently, the questionnaire aimed to uncover the barriers preventing PWD from participating in more sport, while ascertaining the role of attitudes towards the LPG in these barriers. To understand the barriers, 65 Likert items were included that focused on constraints preventing non-active PWD from participating in more sport. Darcy, Lock and Taylor's (2017) constraint items were included in the questionnaire along with items generated by Brown and Pappous' (2018a) study into the decline in sports participation of PWD. The categories of constraints were the following:

- Community/organisation (6 items);

- Perceptions of sporting ability (6 items);

- Time (5 items);

- $\quad$ LPG (8 items);

- Interpersonal (2 items);

- Economic (6 items);

- Intrapersonal (11 items);

- Transport (4 items);

- Sports services and experiences (11 items);

- The role of grassroots sport clubs (6 items).

No responses were received when attempting to pilot the questionnaire. Consequently, to ensure face validity was achieved, the pilot questionnaire was distributed to 5 academics who had experience in MSE sport participation legacy research. Changes suggested by the academics were reviewed and incorporated where appropriate. The large number of items included in the final questionnaire enabled a full exploration of constraints to occur. The sheer number of constraints, however, may have prevented a higher number of responses than was achieved, a problem also experienced by Darcy, Lock and Taylor (2017). The authors felt there was more currency in carrying out an exhaustive review of the constraints while accepting the likelihood this might reduce the number of responses. The authors felt it was important, as this study is exploratory, to understand the essential constraints in the first instance, potentially providing future studies with a reduced set of constraints to explore. To get to a position where consolidated constraints were generated by a factor analysis, the full list of constraints was required. It may be the case the final number of items are reduced as their suitability and fit to measuring constraints to increased sport participation might be revealed to be unsatisfactory. 
The factor analysis therefore enables a robust collection of constraint items for this sample to emerge.

\section{Results}

This section reports the findings from the quantitative data analysis conducted for this study. First, descriptive information about the sample will be presented, followed by descriptive variables about the participants' engagement with the LPG. Finally, the section reports the findings from the exploratory factor analysis into the main constraints limiting more sport participation for this sample.

\section{Participant demographic and LPG engagement information}

Of the 81 participants to complete the questionnaire, $41.98 \%$ had not participated in sport or physical activity within the last 4 weeks (Table 1) and thus can be considered to be inactive (Sport England, 2017). The remaining members of the sample all participated in sport or physical activity for less than 150 minutes a week and were thus not considered 'physically active' according to Sport England's definition of physical activity (Sport England, 2017). The findings from this study are informed mainly from a female perspective, as $70.37 \%$ of the sample are females. Three-quarters of the participants are aged 35 and over $(75.31 \%)$, with a quarter of the sample residing in the south east region of England (25.93\%). The overwhelming majority $(88.89 \%$ ) of participants are from a white British ethnic background. All participants identified as disabled, with mobility impairments (87.65\%) and long-term pain $(64.20 \%)$ being the most common form of impairment amongst the sample.

\section{TABLE 1 HERE}

Table 2 provides an overview of the level of engagement with, and experience of, the LPG by the respondents. Most of the participants followed the LPG by watching television or online coverage (69.14\%), with a little under a fifth of participants attending one of the events live (18.52\%). Only $3.70 \%$ of the sample did not engage with the LPG at all. The participants were fairly evenly split in either being at least not that interested in the LPG before the LPG $(40.74 \%)$ compared to $48.15 \%$ of participants being at least somewhat interested in the LPG before the event. Following the LPG, the most popular consideration amongst participants was to watch more sport for PWD on television $(51.85 \%)$, followed by a consideration of taking part in more sport or exercise (41.98\%). Interestingly, $41.98 \%$ of participants believed either the message of the 'inspirational exploits of Paralympians' (18.52\%) or 'everybody being able to take part in sport regardless of ability' $(23.46 \%)$ was the most important message from the LPG. This would suggest positive perceptions of the LPG 5 years on is possible for a sizeable section of the PWD that completed this questionnaire, despite not participating in enough exercise or sport to be considered 'active'.

\section{TABLE 2 HERE}

\section{A review of the main constraints to more sport participation for non-active PWD}

Constraint items focused on attitudes towards the LPG were not one of the main categories of constraints that limit more sport participation (Table 3). Instead, community and organisational constraints appear to pose a particular challenge to non-active PWD participating in more sport. Impairment effects (Thomas, 1999) appear to be a strong constraint for non-active PWD. This is demonstrated by the strong support for the challenge posed by an individual's impairment on participation in increased sport as well as finding participation opportunities commensurate with the individual's impairment. In terms of sport-specific constraints, sport services and experiences and the role of voluntary sport clubs (VSCs) appear to be the main categories of 
constraints limiting increased sport participation. Attitudes suggesting constraints linked to the LPG do not feature in the top 10 of constraints and the LPG does not appear to be of particular importance in constraining more sport participation for non-active PWD. For PWD that have not participated in sport within the past 12 months, participants disagreed Paralympians are not inspirational $(M=2.54, S D=1.179)$ and the LPG was of no interest $(M=2.33, S D=0.963)$. This suggests, for this particular sample, the hosting of the LPG did not immediately deter interest in participating in more sport, but it was other constraints, such as community and organisational constraints, that were more influential. The portrayal of Paralympians as superhumans was a stronger constraint $(M=3.75, S D=1.152)$ to participation in sport, with inactive PWD finding this off-putting. This suggests the marketing of Paralympians by Paralympic hosts might need to be reviewed as it can act to deter some PWD from participating in more sport.

\section{TABLE 3 HERE}

\section{Factor analysis}

Table 4 presents the results from an exploratory factor analysis on the constraints to more sport participation amongst non-active PWD in England. Factors were extracted using the principal component analysis (PCA) method with oblimin rotation.

\section{TABLE 4 HERE}

When all of the 65 items were run for the PCA, the rotation failed to converge. The solution was only possible once the rotations was set to 100 . Reliability was run for the 65 items and a number of negative correlations were evident. Items with a corrected item-total correlation less than .3 were removed, as Pallant (2016) suggests items with correlations below .3 are unlikely to be unidimensional. To ensure robustness of the components, the decision was taken to only include items which had a loading of at least .60 on a component (Blaikie, 2003). High factor loadings are particularly important when using factor analysis on samples numbering less than 300 (Pallant, 2016). A PCA was performed resulting in 18 items with a loading of at least .60 on a component. The Kaiser-Meyer Olkin measure of sampling adequacy (KMO) for the 18 item solution was a respectable .814 , a KMO score suitable for conducting a PCA (Blaikie, 2003). The mean value of the communalities was .729. Four components had an eigenvalue above 1 and a review of the scree plot supported the use of four components for the data. The categories of constraints for the 18 items were the following:

- Perceptions of sporting ability (2 items);

- Economic (5 items);

- Intrapersonal (1 items);

- Sports services and experiences (8 items);

- The role of grassroots sport clubs (2 items).

The 4 components explained a total of $72.93 \%$ of the total variance. One item, 'Scarce access to adaptable equipment' had more than one loading on the components, with this item loading strongly on component 1 (.636) and then slightly on component 4 (-.314). Due to the relatively small size of the loading on component 4 and the high loading on component 1 , the item was retained as it contributed to the overall strength of component 1 . The item, 'Attitudes of nondisabled people discouraged me from participating in sport' (.595) had a component loading lower than the .60 loading threshold, but as it was only .05 away from .60, discretion was used, and the item was retained. To ensure the identified constraints to more sport participation for non-active PWD were robust, it was necessary to conduct a reliability analysis on the components. Cronbach's Alpha for the 18 items was .906, comfortably exceeding the 
recommended minimum of .70 (Blaikie, 2003). For the individual components, all of the Cronbach Alpha's were above .70 (Table 4). Thus, the components are internally reliable and are unidimensional in the construct being measured (Blaikie, 2003). The 4 components that summarise the main constraints to more sport participation for non-active PWD are:

- Component 1: Sport provision (40.22\% of the total variance)

- Component 2: Economic (16.47\% of the total variance)

- Component 3: Unawareness of how to include PWD in sporting activities $(10.22 \%$ of the total variance)

- Component 4: Access to sport participation opportunities (6.02\% of the total variance)

\section{Interpretation of the components}

The first component relates to the availability and affordability of sports equipment, as well as provision of sport participation opportunities. This component accounted for $40.22 \%$ of the variance and was the most important component identified. It would appear that access and the cost of sports equipment is a significant barrier to participating in more sport for PWD, a finding consistent with other studies (Darcy, Lock \& Taylor, 2017). Allied to this is the provision of sport participation opportunities for PWD, with the perception that sport participation opportunities are not suitable for the specific needs of some PWD (Ives et al., 2019; Jaarsma et al., 2014). The current communication methods of sports organisations might not be effective in reaching non-active PWD, hindering some PWD from participating in more sport (Jaarsma, Haslett \& Smith, 2018).

Component 2, which accounts for $16.47 \%$ amount of the total variance, relates to the economic challenges of participating in more sport. This barrier was also identified by Darcy, Lock and Taylor (2017) in their review of the constraints to sport participation of Australian PWD, and the discovery of this component further supports Darcy, Lock and Taylor's (2017) findings. On average, PWD have lower earnings and wealth compared to non-disabled people (McKnight, 2014), thus the economic implications of participating in more sport is likely to be more acute for some PWD. Furthermore, the UK's austerity measures during the past decade is likely to have compounded this constraint (Brown \& Pappous, 2018b). PWD, more so than people without disabilities, access and make use of public leisure facilities (Kung \& Taylor, 2014), therefore the average agreement that leisure centres and gym are too expensive is likely to have been an important constraint to more sport participation. Despite the efforts of organisations such as the Activity Alliance to make gyms more welcoming to PWD (Activity Alliance n.d.), gyms are still viewed as being too expensive, suggesting the costs of accessing sports facilities is an important and significant barrier affecting PWD's sport participation.

Component 3, which accounts for $10.22 \%$ of total variance, refers to a lack of knowledge of how to include PWD in sport participation activities (Johnson, 2019). This can prevent some PWD from participating in more sport because this lack of understanding can lead to uncomfortable experiences for some PWD. Misinformed and prejudiced views of PWD can also inhibit more participation. One way this can materialise is if VSCs' understanding of disability has been mainly formed through the achievements of Paralympians, and then assuming PWD are equally capable of the elite exploits of Paralympians. This expectation can contrast sharply with the reality for some PWD, like any person trying a new sport for the first time, and therefore the deficit between expectation and reality can be off-putting and act as a deterrent for some PWD (Weed et al., 2009). Ill-informed understanding of disability by nondisabled coaches and VSCs is therefore a source of unease for some PWD and can deter some from increasing their participation sport. 
The items that comprise component 4 are centred around access to sport participation, accounting for $6.02 \%$ of the total variance. Access to sport participation refers to the availability of local sport participation opportunities suitable for the individual's personal requirements, a finding consistent with Darcy, Lock and Taylor (2017). This includes opportunities consistent with the individual's impairment requirements, but also in terms of the level and intensity of sport participation options (Ives et al., 2019).

The barriers to participating in more sport for non-active or less active PWD highlight the complexity involved in changing habits and behaviour change. The components highlight barriers that are beyond the scope for many sports organisations, such as general economic constraints. This highlights the limited influence the demonstration effect can have for sustained participation in sport and as an effective policy intervention, as once the event has concluded many systemic and social challenges may prevent more sport participation from occurring. Table 5 presents the consolidated CMOCs resulting from using the LPG as a policy measure to increase non-active PWD's sport participation.

\section{TABLE 5 HERE}

\section{Discussion}

This research provided an insight into constraints to more sport participation for some nonactive PWD following the LPG. Attitudes towards the LPG, itself, did not appear to prevent more sport participation, but the media representation of Paralympians as superhumans, for this sample, was an ineffective mechanism for increasing participation. The decision to participate in sport is often a complex combination of social, physical, and psychological factors (Rowe, 2015). For PWD, there are added complications in the form of ableist viewpoints in society and sporting organisations (Brown \& Pappous, 2018a), inequal wealth distribution (McKnight, 2014), and inaccessible environments for participating in sport (Jaarsma et al., 2014). With this in mind, the chance of a two-week sporting event, such as the Paralympic Games, being able to significantly alter the sport participation of PWD is unlikely (Brown, 2020). This is especially true for non-active PWD who may be at the precontemplation or contemplation stage of being physically active (Prochaska, DiClemente \& Norcross, 1992; Schwarzer, Lippke \& Luszczynska, 2011). Furthermore, the media representation of Paralympians as superhumans, and the lack of relevancy in terms of lived experiences of disability for PWDs and Paralympians (Howe \& Silva, 2018), exaggerates the difficulty in using the Paralympic Games to increase sport participation. It is argued, therefore, organisations and politicians should not pin their hopes on the demonstration effect increasing the number of PWD participating in sport. To do so would be misguided and divert valuable resources away from initiatives that might be more conducive to encouraging inactive PWD to participate in sport. This is a finding supported by a number of other studies investigating the link between MSEs and sport participation (Weed et al., 2015). Countries should not, therefore, host the Paralympic Games with the primary aim of increasing the number of non-active PWD participating in sport, because this is an unrealistic demand of the event. There may be 'small wins' possible by increasing the leveraging activities associated with the Paralympic Games (Misener, McPherson, McGillivray \& Legg, 2018), though there is a lack of evidence to demonstrate successful leveraging strategies and initiatives at this stage (Pappous \& Brown, 2018). Increased leveraging may still not be enough because, although the Paralympic Games may spur some PWD to participate more frequently in sport or to take up sport, the utility of using inspiration to increase participation is limited for individuals not predisposed to being sporty (Grix \& Carmichael, 2012; Lyle, 2009), precisely the audience likely to be inactive and in need of increasing their sport participation. Furthermore, relying on inspiration alone fails to acknowledge systemic and societal barriers, as well as challenges related to an individual's 
impairment, that can constrain the participation of PWD in sport (Darcy, Lock, \& Taylor, 2017). Systemic and social barriers were found to be more prohibitive to sport participation for non-active PWD than attitudes towards the LPG. The Paralympic Games offers sports a great opportunity to increase their profile among the public, while prompting investment from governments into sport (Brown, 2019), though this is often skewed towards elite sport (Sun, Yan, Mao, Chao \& Jing, 2011). It is important sports organisations do not view the Paralympic Games as a panacea for sports participation: to do so would be folly (Howe \& Silva, 2018). Instead, the Paralympic Games must be leveraged, and grassroots disability sport supported by strategic use of financial and human resources, effective grassroots policies, and consistent monitoring and evaluation. Even if nations achieve this, systemic and social barriers are deeprooted and must be tackled to enable more non-active PWD to participate in more sport. For as long as social and systemic barriers are prevalent in society, hosting a Paralympic Games with the intention to increase the number of non-active PWD participating in sport is likely to be forlorn (Brown, 2020).

Due to the limited number of clubs accessible to PWD in comparison with people without disabilities, VSCs may be geographically further apart from one another. Travel to other VSCs is thus more logistically challenging and costly for PWD participating in most mainstream sports. Inaccessible transport compounds this issue and can be a significant deterrent to commitment to sport participation for some PWD. Increasing the accessibility of transport will therefore increase the transport available to PWD, limiting the impact of this barrier to sport participation. Reducing the number of inaccessible transport options will be costly in the shortterm but may yield a positive economic benefit thereafter. Reduced inaccessible transport will mean more PWD can commute to work, boosting the economy in terms of productivity and tax contributions. It is also important for governments to improve the accessibility to sports venues and stadiums for PWD. Attending sport events may help to increase an individual's interest in sport (Ramchandani, Davies, Coleman, Shibli \& Bingham, 2015) which, in turn, may increase one's commitment to participate in sport. Improvements in transport accessibility would make PWD' attendance at sporting events easier.

The results of this study and work by Brown and Pappous (2018a) produce a refined programme theory of using the Paralympics Games sport as a policy tool for increasing PWD's sport participation:

The effectiveness of the demonstration effect is likely to be felt amongst individuals predisposed to sport; it is unlikely to be able to change behaviours of individuals without an interest in sport. For PWD who are not active or are far-removed from society, the reality of the sport participation experience and access to opportunities is of more importance than inspiration provided by the Paralympic Games. The Paralympic Games is not able to provide sustainable sport participation increases if multiple barriers exist in society. This is because social and systemic barriers are often deep-rooted and are more prominent in the intensity of their ability to constrain sport participation.

\section{Limitations of the research}

The small sample size meant insights garnered from this study were limited to the sample. The findings are therefore not representative of the wider non-active PWD population and provide a descriptive quality for this specific sample. The sampling method relied on gatekeepers and their discretion to support the study, with it impossible using this method to ensure each PWD affiliated to a DPO had an equitable chance of completing the survey. Furthermore, only PWD with access to the internet and affiliation to a DPO could complete the survey. It was not 
possible to obtain findings from non-active PWD who are not associated with a DPO. This population group in society is under researched and may experience the intensity of constraints more acutely than other population groups. Future researchers should aim to provide a voice for this population group, enabling a more diverse and balanced picture of constraints and experiences to emerge. While the limited variability and size of the sample is regrettable, the authors believe the findings have utility. The societal group of non-active PWD can be considered a hard to access population (Bonevski et al., 2014; Rockliffe et al., 2018), thus little is known about this population group. With few sources of evidence for this population, the findings from this study provide an insight into some of the constraining factors for non-active PWD. This is still an advance on the current body of knowledge for this population, which is scarce and underdeveloped.

The questionnaire was unable to receive empirical feedback from a pilot sample, resulting in a large number of constraint items in the final questionnaire. Due to the numerous constraint items, the factor analysis process removed many of the initial items and the associated data, reducing the variety of constraints originally included in the survey (Darcy, Lock \& Taylor, 2017). Though the loss of data is regrettable, the large number of constraint items reflected the exploratory nature of the study. Thus, the decision to include a large number of items was taken in order to not omit potentially important constraints. A criterion of only retaining items with a high loading of .60 on a component enabled robust items to emerge as a result of empirical data.

Disability is not a homogenous population group (Watson, 2002) and the experiences and constraints to more sport participation is likely to be numerous. The findings reported in this study do not provide a granular detail of the different ways in which constraints have prevented more sport participation when considering different impairment types. Obtaining this data would have enhanced the value of the research. Future researchers should strive to understand how constraints can differ between impairment types. Consistent with Darcy, Lock and Taylor (2017), future studies exploring PWD's sport participation constraints should aim to capture the role of socio-demographic variables. The intersection between sport participation constraints and socio-demographic variables such as gender, socio-economic status, sexuality, ethnicity, and culture can provide valuable learnings for future researchers.

Despite the limitations, this research builds on Brown and Pappous' (2018a) study on declining sport participation of PWD in England after the LPG, by providing empirical data on barriers constraining some non-active PWD from participating in more sport since the LPG. This research enables future researchers to understand wider societal and systemic barriers facing PWD, and the utility of using a MSE, in this case the LPG, as a policy measure for increasing sport participation of PWD. This research is therefore an important contribution to the community of scholars operating in the sport policy and Paralympic Games sport participation legacy fields.

\section{Conclusion}

Understanding the barriers constraining some non-active PWD from participating in more sport is important if Paralympic Games hosts are to maximise a potential demonstration effect. A failure to address systemic and social barriers facing PWD shines a light on the limited utility of using the Paralympic Games as a policy intervention. Thus, even if the Paralympic Games is strategically leveraged, sports organisations may still find it difficult or impossible to engage some sections of the disabled population in regular sports participation. Indeed, $41.98 \%$ of participants considered taking part in more sport or exercise. Despite this, these individuals 
have been unable to do so, highlighting the influence and role of barriers to sport participation external to the Paralympic Games. Thus, interest to participate in more sport does not necessarily mean sustainable sports participation will be achieved. Indeed, Active People Survey data highlighted $56.30 \%$ of PWD would like to do more sport participation than they currently do (Active People Interactive, 2017), but some PWD might be prevented from participating in more sport because of these systemic and social barriers.

MSEs can present 'wicked problems' due to the complexity involved in trying to deliver a legacy meeting a myriad of stakeholder requirements and the varying contexts that can impinge on mechanisms firing as intended (Byers, Hayday, \& Pappous, 2019). Governments aiming to increase the sport participation of non-active PWD would do well to focus on local, bottom-up solutions designed and delivered in conjunction with PWD, as these initiatives may be more effective.

\section{Acknowledgments;}

The authors would like to thank the reviewers for their comments and insight in improving the quality of the paper.

\section{Declaration of interest statement}

No potential conflict of interest was reported by the authors.

\section{References}

Active People Interactive. (2017). Analyse the data. Retrieved from http://activepeople.sportengland.org

Activity Alliance. (n.d.). Inclusive Fitness Initiative. Retrieved from http://www.activityalliance.org.uk/how-we-help/programmes/65-inclusive-fitness-initiative

Activity Alliance. (2020). Annual Disability and Activity Survey 2019/20. Retrieved from http://www.activityalliance.org.uk/how-we-help/research/5563-activity-alliance-annualdisability-and-activity-survey

Astbury, B. (2013). Some reflections on Pawson's Science of Evaluation: A Realist Manifesto. Evaluation, 19(4), 383-401. doi: 10.1177\%2F1356389013505039

Astbury, B., \& Leeuw, F. L. (2010). Unpacking Black Boxes: Mechanisms and Theory Building in Evaluation. American Journal of Evaluation, 31(3), 363-381. doi: $10.1177 / 1098214010371972$

BBC Sport (2013). Olympic legacy: Sarah Storey says Paralympics changed attitudes. Retrieved from http://www.bbc.co.uk/sport/0/disability-sport/23450746

Blaikie, N. (2003). Analyzing Quantitative Data: From Description to Explanation. London: SAGE.

Bloyce, D., \& Lovett, E. (2012). Planning for the London 2012 Olympic and Paralympic legacy: a figurational analysis. International Journal of Sport Policy and Politics, 4(3), 361377. doi: 10.1080/19406940.2012.740063

Bogart, K. R. \& Dunn, D. S. (2019). Ableism Special Issue Introduction. Journal of Social Issues, 75(3), 650-664. doi: 10.1111/josi.12354 
Bonevski, B., Randell, M., Paul, C., Chapman, K., Twyman, L., Bryant, J., Brozek, I. \& Hughes, C. (2014). Reaching the hard-to-reach: a systematic review of strategies for improving health and medical research with socially disadvantaged groups. BMC Med Res Methodol, 14(42), 1-29. doi: 10.1186/1471-2288-14-42

Braye, S., Dixon, K. \& Gibbons, T. (2013). 'A mockery of equality': an exploratory investigation into disabled activists' views of the Paralympic Games. Disability \& Society, 28(7), 984-996. doi: 10.1080/09687599.2012.748648

Briant, E., Watson, N. \& Philo, G. (2013). Reporting disability in the age of austerity: the changing face of media representation of disability and disabled people in the United Kingdom and the creation of new 'folk devils'. Disability \& Society, 28(6), 874-889. doi: $10.1080 / 09687599.2013 .813837$

Brittain, I. (2012). British Media Portrayals of Paralympic and Disability Sport. In: O. J. Schantz \& K. Gilbert (Eds.), Heroes or Zeros? The Media's Perceptions of Paralympic Sport. (pp. 105-113). Champaign, Illinois: Common Ground Publishing LLC.

Brittain, I. \& Beacom, A. (2016). Leveraging the London 2012 Paralympic Games: What Legacy for Disabled People? Journal of Sport and Social Issues, 40(6), 499-521. doi: $10.1177 / 0193723516655580$

Brittain, I., Biscaia, R. \& Gérard, S. (2020). Ableism as a regulator of social practice and disabled peoples' self-determination to participate in sport and physical activity. Leisure Studies, 39(2), 209-224. doi: 10.1080/02614367.2019.1694569

Brown, C. (2020, 28 Jan). The Paralympic Games fails to increase disabled people's participation in sport. The Conversation. Retrieved from https://theconversation.com/theparalympic-games-fails-to-increase-disabled-peoples-participation-in-sport-130082

Brown, C. (2019). "I still think we've got mountains to climb”: Evaluating the grassroots sport participation legacy of the London 2012 Paralympic Games for disabled people in England [University of Kent]. https://doi.org/10.1002/cb.1444/abstract.

Brown, C., \& Pappous, A. S. (2018a). "The Legacy Element . . . It Just Felt More Woolly": Exploring the Reasons for the Decline in People With Disabilities' Sport Participation in England 5 Years After the London 2012 Paralympic Games. Journal of Sport and Social Issues, 42(5), 343-368. doi: 10.1177/0193723518781237

Brown, C., \& Pappous, A. S. (2018b). The organisational performance of national disability sport organisations during a time of austerity: a resource dependence theory perspective. International Journal of Sport Policy and Politics, 10(1), 63-78. doi: 10.1080/19406940.2017.1381635

Byers, T., Hayday, E., \& Pappous, A. S. Pappous. (2019). A new conceptualization of mega sports event legacy delivery: Wicked problems and critical realist solution. Sport Management Review. doi: 10.1016/j.smr.2019.04.001

Campbell, F. A. K. (2001). Inciting legal fictions: 'Disability's' date with ontology and the ableist body of the law. Griffith Law Review, 10(1), 42-62.

Christiaens, M. (2018). Towards Mainstreaming: A Principle-Practice Gap in the UK Sports Sector. Coventry University.

Coalter, F. (2007a). London Olympics 2012: 'the catalyst that inspires people to lead more active lives'? Perspectives in Public Health, 127(3), 109-110. doi: 10.1177/1466424007077342 
Coward, D. \& Legg, D. (2011). Vancouver 2010. In: D. Legg \& K. Gilbert (Eds.), Paralympic Legacies. (pp. 131-141). Champaign, Illinois: Common Ground Publishing LLC.

Cross, M. (2013). Demonised, impoverished and now forced into isolation: the fate of disabled people under austerity. Disability \& Society, 28(5), 719-723. doi: 10.1080/09687599.2013.808087

Crow, L. (2014). Scroungers and Superhumans: Images of Disability from the Summer of 2012: A Visual Inquiry. Journal of Visual Culture, 13(2), 168-181. doi: https://doi.org/10.1177/1470412914529109

Darcy, S., Lock, D., \& Taylor, T. (2017). Enabling Inclusive Sport Participation: Effects of Disability and Support Needs on Constraints to Sport Participation. Leisure Sciences, 39(1), 20-41. doi: 10.1080/01490400.2016.1151842

Department for Culture, Media \& Sport (DCMS). (2010). London 2012: A legacy for disabled people. London: Department for Culture, Media \& Sport. Retrieved from http://webarchive.nationalarchives.gov.uk

Department for Culture, Media \& Sport (DCMS). (2008). Before, during and after: making the most of the London 2012 Games. London: Department for Culture, Media \& Sport. Retrieved from http://webarchive.nationalarchives.gov.uk

ECORYS. (2018). Mapping on access to sport for people with disabilities: A report to the European Commission. Retrieved from https://ec.europa.eu/sport/sites/sport/files/mapping_access_to_sport_for_people_with_disabili ites_final.pdf

English Federation of Disability Sport (EFDS) (2013). EFDS Report of the Sport and Recreation Alliance's Sports Club Survey 2013 A baseline understanding of the provision for disabled people among UK sports clubs. EFDS. Retrieved from http://www.activityalliance.org.uk/how-we-help/research/1909-efds-report-on-sport-andrecreation-alliances-sports-club-survey-2013

Gibson, O. (2012, 13 Aug). London 2012: invest in elite sport for grassroots legacy, says Lord Coe. The Guardian. Retrieved from https://www.theguardian.com/sport/2012/aug/13/london2012-lord-coe-investment-elite-sport

Gibson, O. (2009, October 21). Legacy is the story for 2012, says Coe. The Guardian. Retrieved from https://www.theguardian.com/uk/2009/oct/21/sebastian-coe-london-2012olympics-legacy

Gilbert, K. and Schantz, O. J. (2012). An Implosion of Discontent. In: O. J. Schantz \& K. Gilbert (Eds.), Heroes or Zeros? The Media's Perceptions of Paralympic Sport. (pp. 225-237). Champaign, Illinois: Common Ground Publishing LLC.

Grix, J., \& Carmichael, F. (2012). Why do governments invest in elite sport? A polemic. International Journal of Sport Policy and Politics, 4(1), 73-90. doi: 10.1080/19406940.2011.627358

HM Government. (2015). Sporting Future: a new strategy for an active nation. London: Cabinet Office. Retrieved from https://www.gov.uk/government/publications/sporting-futurea-new-strategy-for-an-active-nation 
Howe, P. D. (2011). Cyborg and Supercrip: The Paralympics Technology and the (Dis)empowerment of Disabled Athletes. Sociology, 45(5), 868-882. doi: $10.1177 / 0038038511413421$

Howe, P. D. \& Silva, C. F. (2018). The fiddle of using the Paralympic Games as a vehicle for expanding [dis]ability sport participation. Sport in Society: Cultures, Commerce, Media, Politics, 21(1), 125-136. doi: 10.1080/17430437.2016.1225885

Hughes, K. H. (2013). Sport mega-events and a legacy of increased sport participation: An Olympic promise or an Olympic dream? (Doctoral thesis). Leeds: Leeds Metropolitan University.

Ives, B., Clayton, B., Brittain, I. \& Mackintosh, C. (2019). 'I'll always find a perfectly justified reason for not doing it': challenges for disability sport and physical activity in the United Kingdom. Sport in Society, 1-19. doi: 10.1080/17430437.2019.1703683

Jaarsma, E. A., Dijkstra, P. U., Geertzen, J. H. B. \& Dekker, R. (2014). Barriers to and facilitators of sports participation for people with physical disabilities: A systematic review. Scandinavian Journal of Medicine \& Science in Sports, 24(6), 871-881. doi: $10.1111 / \mathrm{sms} .12218$

Jaarsma, E., Haslett, D. \& Smith, B. (2018). Improving Communication of Information About Physical Activity Opportunities for People With Disabilities. Adapted Physical Activity Quarterly, 36(2), 185-201. doi: 10.1123/apaq.2018-0020

Johnson, E. (2019). Delivering activity to disabled people: The workforce perception gap. Retrieved from http://www.activityalliance.org.uk/assets/000/002/641/Activity_Alliance_Deliverer_Percepti ons_Research_Report_Final_Accessible_PDF_report_original.pdf?1548778930

Johnson, E., \& Spring, E. (2018). The Activity Trap: Disabled people's fear of being active. Retrieved from http://www.activityalliance.org.uk/how-we-help/research/the-activity-trap

Kung, S. P., \& Taylor, P. (2014). The use of public sports facilities by the disabled in England. Sport Management Review, 17(1), 8-22. doi: 10.1016/j.smr.2013.08.009

Lyle, J. (2009). Sporting success, role models and participation: A policy related review. Edinburgh: $\quad$ Sportscotland. Retrieved from https://sportscotland.org.uk/documents/resources/sportingsuccessrolemodelsandparticipation. pdf

McKnight, A. (2014). Disabled people financial histories: uncovering the disability wealthpenalty. Centre for Analysis of Social Exclusion, CASE/181. Retrieved from http://sticerd.lse.ac.uk/dps/case/cp/casepaper181.pdf

Misener, L., Darcy, S., Legg, D. \& Gilbert, K. (2013). Beyond Olympic Legacy: Understanding Paralympic Legacy Through a Thematic Analysis. Journal of Sport Management, 27, 329-341. doi: $10.1123 /$ jsm.27.4.329

Misener, L., McPherson, G., McGillivray, D. \& Legg, D. (2018). Leveraging Disability Sport Events: Impacts, Promises, and Possibilities. Abingdon, Oxon: Routledge.

Office for Disability Issues. (2011). London 2012: a legacy for disabled people. London, United Kingdom: Office for Disability Issues. Retrieved from 
http://webarchive.nationalarchives.gov.uk/20130703134200/http://odi.dwp.gov.uk/docs/wor/l eg/legacy-full.pdf

Pallant, J. F. (2016). SPSS Survival Manual: A Step by Step Guide to Data Analysis Using IBM SPSS (6th ed.). New York: Allen \& Unwin.

Pappous, A. S. \& Brown, C. (2018). Paralympic Legacies: A Critical Perspective. In: I. Brittain \& A. Beacom. (Eds.), The Palgrave Handbook of Paralympic Studies. (pp 647-664). Palgrave Macmillan, London.

Pawson, R. (2013). The science of evaluation: A realist manifesto. London: Sage.

Pawson, R., \& Tilley, N. (2004). Realist Evaluation. Retrieved from http://www.communitymatters.com.au/RE_chapter.pdf

Pawson, R., \& Tilley, N. (1997). Realistic evaluation. London: Sage.

Payne, W., Reynolds, M., Brown, S., \& Fleming, A. (2003). Sports role models and their impact on participation in physical activity: a literature review. Victoria: VicHealth.

Perreault, S. \& Vallarand, R. J. (2007). A test of self-determination theory with wheelchair basketball players with and without disability. Adapt Phys Act Q, 24(4), 305-316. doi: 10.1123/apaq.24.4.305

Prochaska, J. O., DiClemente, C. C. \& Norcross, J. C. (1992). In search of how people change: Applications to addictive behaviors. American Psychologist, 47(9), 1102-1114.

Purdue, D. E. J. \& Howe, P. D. (2012). Empower, inspire, achieve:(dis)empowerment and the Paralympic Games. Disability \& Society, 27(7), 903-916. doi: 10.1080/09687599.2012.695576

Ramchandani, G., Davies, L. E., Coleman, R., Shibli, S. and Bingham, J. (2015). Limited or lasting legacy? The effect of non-mega sport event attendance on participation. European Sport Management Quarterly, 15(1), 93-110. doi: 10.1080/16184742.2014.996583

Rockliffe, L., Chorley, A. J., Marlow, L. A. V. \& Forster, A. S. (2018). It's hard to reach the "hard-to-reach": the challenges of recruiting people who do not access preventative healthcare services into interview studies. International Journal of Qualitative Studies on Health and Well-being, 13(1), 1-12. doi: 10.1080/17482631.2018.1479582

Rowe, N. (2015). Sporting capital: a theoretical and empirical analysis of sport participation determinants and its application to sports development policy and practice. International Journal of Sport Policy and Politics, 7(1), 43-61. doi: 10.1080/19406940.2014.915228

Scelza, W. M., Kalpakjian, C. Z., Zemper, E. D. Tate, D. G. (2005). Perceived barriers to exercise in people with spinal cord injury. Am J Phys Med Rehabil, 84(8), 576-583. doi: 10.1097/01.phm.0000171172.96290.67

Schwarzer, R., Lippke, S. \& Luszczynska, A. (2011). Mechanisms of Health Behavior Change in Persons With Chronic Illness or Disability: The Health Action Process Approach (HAPA). Rehabilitation Psychology, 56(3), 161-170. doi: 10.1037/a0024509

Silva, C. F. \& Howe, P. D. (2012). The (In)validity of Supercrip Representation of Paralympian Athletes. Journal of Sport and Social Issues, 36(2), 174-194. doi: 10.1177/0193723511433865

Sport and Recreation Alliance. (2013). Sports Club Survey 2013. Retrieved from https://www.sportandrecreation.org.uk/policy/research-publications/sports-club-survey-2013 
Sport England (2017). Active Lives Survey 2015-16: Year 1 Report. London: Sport England. Retrieved from https://www.sportengland.org/media/11498/active-lives-survey-yr-1report.pdf

Sport England. (2016). Towards an active nation: Strategy 2016-2021. London: Sport England. Retrieved from https://direct.sportengland.org/active-nation/our-strategy/

Sun, S., Yan, R., Mao, A., Chao, L. \& Jing, T. (2011). China and the development of sport for persons with a disability, 1978-2008: a review. Sport in Society: Cultures, Commerce, Media, Politics, 14(9), 1192-1210. doi: 10.1080/17430437.2011.614776

Thomas, C. (1999). Female Forms: Experiencing and Understanding Disability. Philadelphia, Pennsylvania: Open University Press.

Thomas, N. \& Smith, A. (2003). Preoccupied with Able-Bodiedness? An Analysis of the British Media Coverage of the 2000 Paralympic Games. Adapted Physical Activity Quarterly, 20(2), 166-181. doi: 10.1123/apaq.20.2.166

Tilley, N. (2000). Realistic evaluation: An overview. Paper presented at the Founding Conference of the Danish Evaluation Society, September 2000. Retrieved from http://healthimpactassessment.pbworks.com/f/Realistic+evaluation+an+overview++UoNT+England+-+2000.pdf

Veal, A. J., \& Darcy, S. (2014). Research Methods in Sport Studies and Sport Management: A Practical Guide. Oxon: Routledge.

Watson, N. (2002). Well, I know this is going to sound very strange to you, but I don't see myself as a disabled person: Identity and disability. Disability \& Society, 17(5), 509-527. doi: $10.1080 / 09687590220148496$

Weed, M. (2016). Should we privilege sport for health? The comparative effectiveness of UK Government investment in sport as a public health intervention. International Journal of Sport Policy and Politics, 8(4), 559-576. doi: 10.1080/19406940.2016.1235600

Weed, M. (2009). The potential of the demonstration effect to grow and sustain participation in sport. Review Paper for Sport England. Canterbury Christ Church University: Centre for Sport, Physical Education \& Activity Research (SPEAR).

Weed, M. \& Dowse, S. (2009). A missed opportunity waiting to happen? The social legacy potential of the London 2012 Paralympic Games. Journal of Policy Research in Tourism, Leisure and Events, 1(2), 170-174. doi: 10.1080/19407960902992241

Weed, M., Coren, E., Fiore, J., Mansfield, L., Wellard, I., Chatziefstathiou, D., \& Dowse, S. (2009). A Systematic Review of the Evidence Base for Developing a Physical Activity and Health Legacy from the London 2012 Olympic and Paralympic Games. Department of Health.

Weed, M., Coren, E., Fiore, J., Wellard, I., Chatziefstathiou, D., Mansfield, L., \& Dowse, S. (2015). The Olympic Games and raising sport participation: a systematic review of evidence and an interrogation of policy for a demonstration effect. European Sport Management Quarterly, 15(2), 195-226. doi: 10.1080/16184742.2014.998695

Williamson, C., Baker, G., Mutrie, N., Niven, A. \& Kelly, P. (2020). Get the message? A scoping review of physical activity messaging. International Journal of Behavioral Nutrition and Physical Activity, 17(51), 1-15. doi: 10.1186/s12966-020-00954-3 
${ }^{\mathrm{i}}$ In 2010, the Conservative Party won the most seats in the 2010 UK General Election but fell short of the number of seats required to form a majority government. The Conservative Party, as the largest party in the UK, formed a Coalition government with the Liberal Democratic Party, the third largest party. This was the first time since the Second World War the UK had a ruling Coalition government. Readers interested in learning more about the Coalition government are recommended to consult the following $\mathrm{BBC}$ article: http://news.bbc.co.uk/1/hi/uk_politics/election_2010/8676607.stm

ii Sport England is the non-governmental body responsible for grassroots sport in England.

iii Sport England invested $£ 91,477,960$ into forty-two national governing bodies of sport in receipt of 2013-17 whole sport funding for grassroots disability sport participation targets (Brown, 2019). Please see Brown (2019) for a breakdown of the investment.

iv The English Federation of Disability Sport (EFDS) changed its name to the Activity Alliance in 2018.

$\checkmark$ The Active People Survey was a national survey tracking the sport participation of adults (16+) in England. The survey ran from 2005-2016, subsequently being replaced by the Active Lives Survey. The Active Lives survey places increased emphasis on capturing data on individuals' wider physical activity as well as their sports participation. This survey has been in operation since November 2015 and measures physical activity and sports participation in England for individuals aged 5+. For more information, please consult the dedicated Sport England Active Lives Survey online portal: https://activelives.sportengland.org/ 